\title{
Prevention interventions for human immunodeficiency virus in drug-using women with a history of partner violence
}

This article was published in the following Dove Press journal:

Substance Abuse and Rehabilitation

28 January 2012

Number of times this article has been viewed

\author{
Jamila K Stockman' \\ Natasha Ludwig-Barron' \\ Monica A Hoffman ${ }^{2}$ \\ Monica D Ulibarri ${ }^{3}$ \\ Typhanye V Penniman Dyer ${ }^{4}$ \\ 'Division of Global Public \\ Health, Department of Medicine; \\ ${ }^{2}$ Department of Communication \\ and Science Studies; ${ }^{3}$ Department of \\ Psychiatry, University of California, \\ San Diego, La Jolla, CA; ${ }^{4}$ Department \\ of Epidemiology and Biostatistics, \\ University of Maryland School of \\ Public Health, College Park, MD, USA
}

\begin{abstract}
The intersecting epidemics of human immunodeficiency virus (HIV) and partner violence disproportionately affect women who use drugs. Despite accumulating evidence throughout the world linking these epidemics, HIV prevention efforts focused on these synergistic issues as well as underlying determinants that contribute to the HIV risk environment (eg, housing instability, incarceration, policing practices, survival sex) are lacking. This article highlights selected behavior change theories and biomedical approaches that have been used or could be applied in HIV prevention interventions for drug-using women with histories of partner violence and in existing HIV prevention interventions for drug-using women that have been gender-focused while integrating histories of partner violence and/or relationship power dynamics. To date, there is a paucity of HIV prevention interventions designed for drug-using women (both in and outside of drug treatment programs) with histories of partner violence. Of the few that exist, they have been theory-driven, culture-specific, and address certain aspects of gender-based inequalities (eg, gender-specific norms, relationship power and control, partner violence through assessment of personal risk and safety planning). However, no single intervention has addressed all of these issues. Moreover, HIV prevention interventions for drug-using women with histories of partner violence are not widespread and do not address multiple components of the risk environment. Efficacious interventions should target individuals, men, couples, and social networks. There is also a critical need for the development of culturally tailored combination HIV prevention interventions that not only incorporate evidence-based behavioral and biomedical approaches (eg, microbicides, pre-exposure prophylaxis, female-initiated barrier methods) but also take into account the risk environment at the physical, social, economic and political levels. Ultimately, this approach will have a significant impact on reducing HIV infections among drug-using women with histories of partner violence.
\end{abstract}

Keywords: human immunodeficiency virus, acquired immune deficiency syndrome, interventions, drugs, women, partner violence, gender-based inequalities

\section{Introduction}

Human immunodeficiency virus (HIV) continues to pose a serious and disproportionate threat to the health and well-being of women worldwide, with women making up over half of persons living with HIV/acquired immune deficiency syndrome (AIDS). ${ }^{1}$ In the US, women account for more than one quarter of all new HIV diagnoses. ${ }^{1,2}$ Among US women, the most common modes of HIV transmission are heterosexual contact, which accounts for $72 \%$ of new HIV infections, and injection drug use, which accounts for $26 \%$ of new infections. ${ }^{2}$ In drug-using women worldwide, HIV infections continue to increase, especially among injecting drug users in Eastern Europe, South America, and $\mathrm{Asia}^{3,4}$ and crack-cocaine users in the US and other countries. ${ }^{5,6}$ 
Women who use drugs are at risk for HIV infection through unprotected sex and unsafe injections, both of which may be influenced by gender-based inequalities, such as physical and sexual violence by intimate or paying sex partners, relationship power differentials, and reliance on partners for procurement of drugs. ${ }^{5-10}$ Among drug-using women, the prevalence of physical and sexual intimate partner violence (IPV) is 3-5 times higher compared with women who do not use drugs. ${ }^{6,8}$ The routine activities and behaviors associated with illicit drug use (buying, selling, and obtaining drugs; visiting shooting galleries; conflicts around splitting and sharing drugs with sex partners; and being forced to supply drugs for main partners through sex trading $)^{5,11,12}$ may lead to or increase partner violence. Furthermore, partner violence and drug use often occur in a cyclical relationship in which substance abuse increases the risk for future physical and sexual violence, and physical and sexual violence increases the risk of subsequent substance abuse. ${ }^{13}$

El-Bassel et a $1^{8}$ recently provided suggestions for cognitive behavioral components that focus on the victim and may be beneficial for HIV risk reduction interventions integrating HIV and IPV. These are equally applicable for partner violence and include: raising awareness of the interpersonal contexts and triggers of HIV risk behavior and partner violence, for example, considering how requests for condom use in an intimate relationship may trigger sexual and physical violence and how fear and experience of partner violence prevents some drug-using women from protecting themselves from HIV; developing an accurate appraisal of risk of HIV and partner violence as well as increasing safety planning, problem solving, and sexual communication skills to negotiate safer relationships with their partners; and increasing access and utilization of services and bolstering informal social support to help women establish and maintain relationship safety. ${ }^{8}$ Additionally, there are underlying determinants that contribute to the HIV risk environment for drug-using women with histories of partner violence that should be considered in intervention development.

The risk environment framework has received increasing attention in adequately characterizing the risk for HIV infection in at-risk populations. It asserts that four types of environmental influences, ie, physical, social, economic, and political, interact at the micro and macro levels to explain HIV transmission in at-risk populations. The micro-risk environment focuses on personal decisions and the influence of community-level norms and practices, while the macro-risk environment encompasses structural factors, such as laws, military actions, economic conditions, and wider cultural beliefs. ${ }^{14}$ Table 1 summarizes the HIV risk environment for drug-using women with histories of partner violence. Micro-level determinants include homelessness and housing instability, incarceration, childhood sexual abuse, police mistreatment and arrest, sex in exchange for drugs, shelter, and/or money, and access to drug treatment. Macro-level

Table I Human immunodeficiency virus risk environment model for drug-using women with histories of partner violence

\begin{tabular}{lll}
\hline Risk environment & Micro-environmental & Macro-environmental \\
\hline Physical & - Homelessness & - Drug trafficking and distribution routes \\
& - Housing instability & - Deportation \\
& - Prisons/incarceration & - Geographical shifts in population \\
- Drug use locations & \\
- Relationship and network dynamics & - Stigma and discrimination \\
& - Peer norms & - Social marginalization \\
& - Physical/sexual violence & - Exposure to conflict or disasters \\
& - Childhood sexual abuse & - Ethnic or racial disparities \\
& - Community attitudes & - Gender inequalities \\
& - Local policing practices (eg, police mistreatment, arrests) & - Social and cultural norms \\
& - Cost of male and female condoms, syringes, medication & - Scarcity of health and social services revenue and spending \\
& - Few income generation and employment opportunities & - Employment practices \\
& - Survival sex work & - Economic development \\
& - Cost of health care & \\
Economic & Ensuring widespread coverage of: & Laws and policies governing: \\
& - HIV/STI testing and counseling & - Protection of human and health rights \\
& - Housing assistance for drug users, abused women & - Violence against women \\
& - Sterile needles and syringes & - Sossession of drugs \\
& - Drug treatment & - Free highly active antiretroviral therapy coverage \\
& - Male and female condoms & - Drug treatment
\end{tabular}

Content for the risk environment model adapted from Rhodes and Simic ${ }^{14}$ and Strathdee et al. ${ }^{15}$

Abbreviations: HIV, human immunodeficiency virus; STI, sexually transmitted infections. 


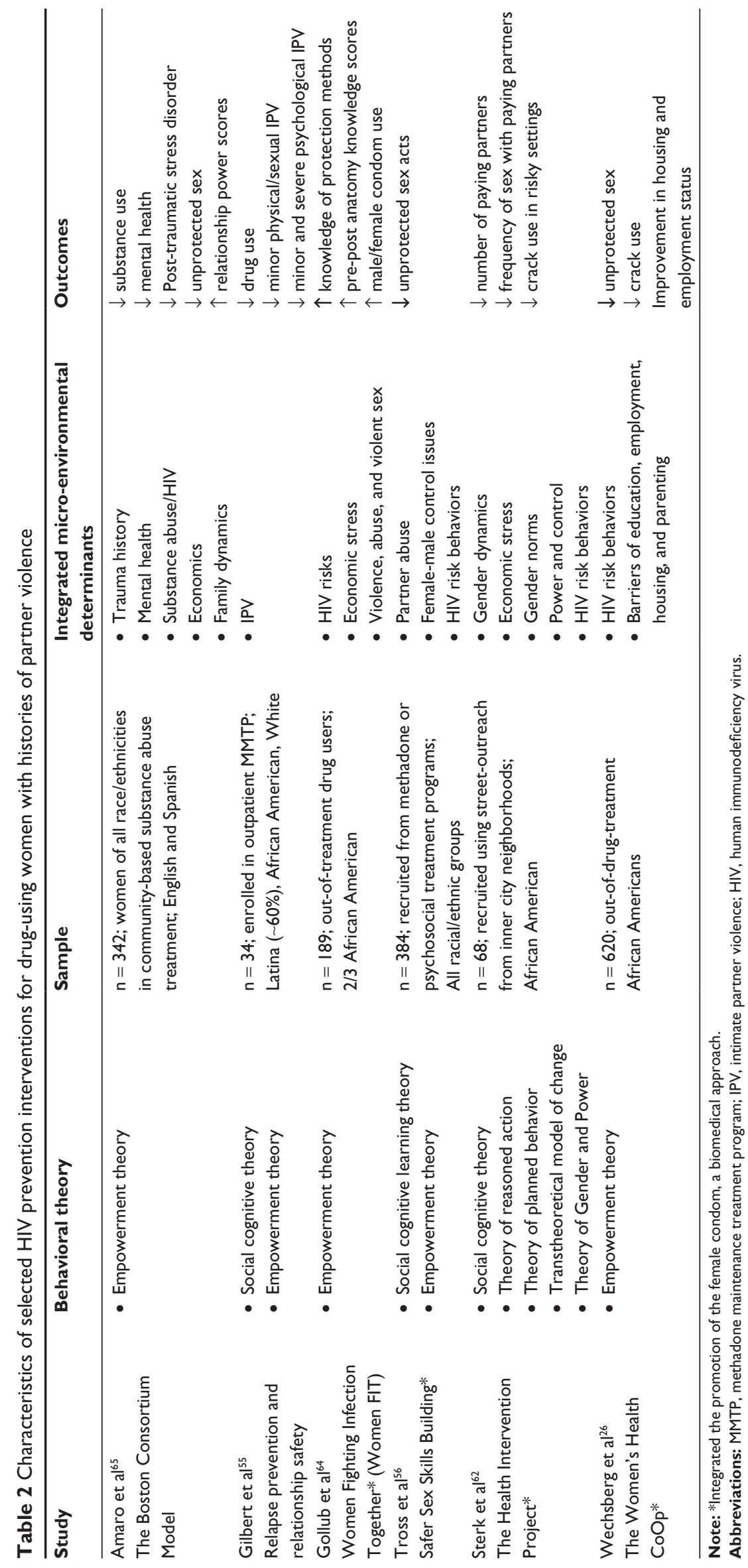


determinants include deportation, social marginalization, stigma, social and cultural norms, lack of government programs to fund specialized health services, and lack of public policies that address violence against women. ${ }^{15}$

While there have been numerous theory-based HIV prevention interventions that have demonstrated efficacy in reducing sexual and drug risk behaviors of drug-using women with or at risk for HIV, few intervention studies have examined outcomes of interventions targeting drugusing women who are also victims of partner violence, with attention to underlying determinants at the micro- and macro-environmental levels. In this article, we present behavior change theories and biomedical approaches that have either been integrated or could be integrated into HIV prevention intervention efforts for drug-using women with histories of partner violence. We also highlight existing HIV prevention interventions for drug-using women that have been gender-focused while integrating histories of partner violence and/or relationship power dynamics, and whether or not micro-level or macro-level environmental determinants were addressed. We conclude with recommendations for future HIV prevention interventions that can comprehensively address the intersecting epidemics of HIV, drug use, and partner violence.

\section{Theoretical and biomedical considerations}

Historically, a number of behavioral theories have been used either individually or concurrently to guide the development and implementation of HIV prevention interventions. Focusing on drug-using women with histories of partner violence, the following theories concerning violence against women and power and control dynamics capture genderbased inequalities: 1) the Theory of Gender and Power, 2) Empowerment theory, and 3) the Substance Abuse, Violence, and AIDS (SAVA) syndemic theory.

\section{Theory of Gender and Power}

The Theory of Gender and Power provides a conceptual framework of social influences that compromise women's health and autonomy, ie, division of labor, division of power, and the structure of cathexis. ${ }^{16}$ Division of labor refers to the unequal allocation of women to certain occupations including "unpaid nurturing work" such as child care, housework, and caring for the sick and elderly, often resulting in an economic imbalance whereby women have to rely on men financially. Applied to HIV prevention, women relying on men for drugs and shelter often resort to exchanging sex for survival and increase the risk of engaging in risky sexual behaviors at the request of their male partners or clients. ${ }^{11}$ Division of power recognizes the power imbalances in heterosexual relationships that contribute to male authority, control, and coercion over women. Within this structure, women at increased risk for HIV are those with histories of sexual or physical abuse, low self-assertiveness and self-efficacy, relationships with a high-risk steady partner or a partner who disapproves of HIV prevention methods, and limited perceived control in their relationships. ${ }^{17}$ Applied to drug-using women, men are often the initiators of drug use by women, play a role in the progression of their drug use, and are often involved in drug-related crimes, including sex work, which can increase a women's risk for HIV. ${ }^{18-20}$ When multiplicities of risks are encountered (eg, drug use, sex work, IPV), women often lack the ability to negotiate safer sex practices. Cathexis refers to society's gender-approved norms and expectations for appropriate sexual behavior. It has been noted that the Theory of Gender and Power, in isolation, implies an intervention with unstructured discussion among the participants about gender and power issues and about how women cope with these issues in their daily lives. ${ }^{21}$ When developing HIV prevention interventions for drug-using women, researchers often target characteristics related to the divisions of labor and power.

\section{Empowerment theory}

Several empowerment theories have emerged in the literature; however, public health researchers and practitioners use the broader concept of empowerment as an enabling process through which individuals, communities, or organizations gain mastery over their lives by transforming their social and political environment to improve equity and quality of life. ${ }^{22,23}$ Empowerment processes and outcomes are typically analyzed at the individual, organizational, and community level, with individual empowerment defined as a person's perceived control of their life, their critical awareness of their social context, and their political efficacy and participation in change. ${ }^{24,25}$ HIV research grounded in women's empowerment theory recognizes that a woman's ability and willingness to protect herself from HIV is influenced by her sense of empowerment developed through daily interactions and experiences in her social contexts. ${ }^{26}$ Empowerment-oriented interventions aim to enhance wellness and reduce barriers faced by target populations, provide opportunities for participants to acquire knowledge and skills, and engage professionals as collaborators, instead of authoritative experts. ${ }^{27}$ Empowerment strategies have shown positive outcomes (eg, improved negotiation 
skills, sexual risk reduction) among drug-using women who engage in sex work and women who have experienced IPV. ${ }^{28-30}$ The potential of HIV prevention interventions that incorporate empowerment strategies to known risk factors (eg, drug use, histories of partner violence), not only have the opportunity to address the underlying determinants and high-risk behaviors but also have sustainable effects through increased peer and social support.

\section{Substance Abuse, Violence, and AIDS syndemic theory}

The SAVA syndemic theory has not been applied to drugusing women with histories of partner violence yet, but it could serve as a comprehensive theoretical framework. Addressing the dynamic physical and psychological interplay of substance abuse, violence, and AIDS, the SAVA syndemic theoretical framework promotes a holistic approach to reducing HIV. ${ }^{31,32}$ SAVA syndemic theory suggests epidemics and conditions, such as violence, substance abuse, and HIV prevalence, are synergistic and cannot be addressed individually. In marginalized communities, syndemics reflect the interplay of broader political, economic, and social factors (eg, poor health care, racism, unemployment, migration), which can further complicate an individual's attempt to engage in healthy behaviors. ${ }^{33,34}$ For example, an injection drug user may be aware of the adverse consequences of sharing a needle; however, their HIV prevention methods may become a secondary priority because self-medication for psychological symptoms of violence and trauma or avoiding withdrawal symptoms may take precedence. Similarly, a woman experiencing IPV may avoid requesting condom use during sexual intercourse, fearing the possibility of a violent reaction from her abusive partner. Development of HIV prevention interventions should integrate harm reduction best practices, empowerment strategies, and behavioral strategies to protect women against HIV.

\section{Biomedical approaches}

Emerging biomedical approaches include medical interventions to block HIV infection, decrease infectiousness, or reduce the risk of HIV infection, and can be conceptualized within various behavior change theories, as well as the risk environment framework. Selected interventions under investigation or proven to be efficacious that may be an eventual reality for drug-using women are antiretroviral drugs as pre-exposure prophylaxis, microbicides, and female-initiated barrier methods. Such methods may be more feasible for vulnerable populations, such as drug-using women with histories of violence, who may lack the ability to engage in safer sex behaviors, including male condom use, which is often controlled by the male sex partner. Moreover, the Fiscal Year 2012 TransNational Institutes of Health Plan for HIV-Related Research emphasizes the prevention of new HIV infections as a top priority through a combination of various biomedical, behavioral, and structural interventions. ${ }^{35}$ Nevertheless, this is an area that needs research, if discovered vulnerable women who use these techniques may be exposed to further violence.

\section{Oral antiretroviral prevention}

Antiretroviral HIV prophylaxis has been efficacious in reducing mother-to-child HIV transmission and has since been explored as an effective method against other routes of transmission. ${ }^{36}$ Pre-exposure prophylaxis uses antiretroviral therapy in anticipation of HIV exposure from known or unknown sources with the aim of preventing HIV acquisition or, at least, altering the natural course of infection to attenuate disease progression, reduce morbidity, and/or decrease infectiousness. ${ }^{37}$ Pre-exposure prophylaxis is a promising prevention strategy consisting of oral administration of continuous (daily) antiretroviral medication by HIV-negative individuals before potential exposure and throughout periods of risk. ${ }^{38}$

An advantage of pre-exposure prophylaxis is that it can be used independently of sexual practices and other risk behaviors. ${ }^{36}$ Medical prophylaxis is standard for the prevention of many other infections such as malaria, but its use is experimental for HIV prevention. Multiple lines of evidence, including animal data, human clinical trials, and mathematical models, suggest that pre-exposure prophylaxis might be effective in preventing HIV among high-risk populations. ${ }^{37-39}$ Candidates for use as oral pre-exposure prophylaxis agents have largely been selected from drugs that are currently approved for treatment of HIV infection. The characteristics that make for an effective pre-exposure prophylaxis drug overlap with those for treatment drugs, including good tolerability and safety, low pill burden, once-daily dosing, long half-life, high potency, and a good resistance profile (ie, the HIV virus does not rapidly develop resistance to the drug or broad cross-resistance with other drugs). ${ }^{38}$ Recent studies have shown pre-exposure prophylaxis to be effective among heterosexual men and women, although important questions remain about which high-risk populations (eg, drug users, ethnic minorities) would benefit most. ${ }^{40}$

\section{Microbicides}

Microbicides are chemical agents in the form of creams, gels, and suppositories, applied topically within the vagina 
or rectum before sexual intercourse in order to prevent HIV and sexually transmitted infections (STIs). ${ }^{41,42}$ The majority of microbicide candidates currently under testing are formulated with antiretroviral drugs. Researchers are exploring their daily use as a gel and other formulations, such as a ring, that in theory could be inserted once a month. ${ }^{42}$ Tenofovir, a nucleotide analog reverse transcriptase inhibitor used in HIV/AIDS therapy, represents the most advanced candidate. ${ }^{43}$ It also represents the first vaginal microbicide proven to be safe and efficacious in the primary prevention of HIV in women. A $1 \%$ vaginal gel formulation of tenofovir, delivered into the vaginal vault up to 12 hours before and within 12 hours after intercourse, reduced HIV acquisition by nearly $40 \%$ overall in the recently completed CAPRISA (Centre for the AIDS Programme of Research in South Africa) 004 Phase IIb HIV prevention trial, which involved 889 South African women. ${ }^{43,44}$

Several other clinical trials are underway investigating anti-HIV microbicides based on non-nucleoside reverse transcriptase inhibitors, such as dapivirine. Other classes of microbicides involve specific entry inhibitors, including gp120 blockers, gp41 blockers, and CCR5 antagonists, integrase inhibitors, protease inhibitors, and a combination of active ingredients. ${ }^{42,43}$ Although the commercial availability of microbicides is likely to be several years away, strategies for regulatory approval and successful product launch should be initiated to address the cultural and educational issues that will be essential to ensure that they are used correctly and in accordance with other prevention strategies. ${ }^{45}$ Drug-using women have expressed a high level of interest in using microbicides with primary and paying partners. ${ }^{46,47}$ However, in one study, drug-using women's concern about a paying partner's violent response to the suggestion of risk reduction measures including microbicides was inversely related to the likelihood of microbicide use. ${ }^{47}$

\section{Female-initiated barrier methods}

The female condom was approved by the US Food and Drug Administration in 1993 as a method to protect against unplanned pregnancy as well as STIs, including HIV/ AIDS. ${ }^{48}$ It remains the only female-initiated biomedical method available for prevention of HIV transmission. ${ }^{36}$ The female condom provides a physical barrier that prevents exposure to genital secretions containing HIV, such as semen and vaginal fluid. ${ }^{49}$ It has received widespread attention both for its physical prevention capabilities and for its symbolic implications in reversing power dynamics in heterosexual relationships. ${ }^{50}$
With the support of the Joint United Nations Programme on HIV/AIDS (UNAIDS), public and private funders, and manufacturers, more than 90 developing countries have introduced the female-initiated barrier method through public distribution, social marketing campaigns or commercial outlets. ${ }^{51}$ In several countries that have actively promoted its use, such as South Africa, Brazil, Ghana, and Zimbabwe, steadily increasing female condom sales to the government suggest that effective programs can generate demand. ${ }^{50}$ However, in Western countries, uptake of the methods has been lower than expected due to user acceptability, its high cost, concerns about the lack of data on its efficacy to prevent HIV infection, and indirect evidence of provider bias against the method. ${ }^{36,49,51}$

Assessment of female condom use among drug-using women with histories of partner violence has been limited. In one study of drug-using women introduced to the female condom at a baseline study visit, a significant proportion of women reported using the female condom at 1-3 weeks of follow-up and many women preferred the female condom to the male condom in terms of overall satisfaction. ${ }^{52}$ And in another study, intentions to use female condoms among drug-involved female sex workers was found to be related to positive attitudes towards both the male and female condom, perceptions of her sexual partner's reaction to the female condom, her perceived safer sex communication skills, and her social network. ${ }^{53}$ Advantages for female-initiated barrier methods for drug-using women include the potential for prior placement and use of a female condom without being noticed by a drunk or "high" partner and the "contraceptive justification" to partners or clients initially unwilling to accede to use of a female barrier. ${ }^{54}$ Recognition that gender-based inequalities, such as IPV and relationship power dynamics, continue to fuel the spread of HIV among women, especially in resource-poor countries, reinforces the need to promote the only female-initiated barrier method for preventing both pregnancy and STIs/HIV.

\section{HIV prevention interventions}

Since the recognition of the multiplicity of risks drug-using women face, few intervention trials have been specifically designed to address gender-based inequalities such as genderbased violence and relationship power for drug-using women. Recognizing that there are a number of best evidence interventions by the Centers for Disease Control and Prevention that are geared specifically towards women, this section is not intended to be a comprehensive review of the literature regarding woman-focused interventions nor HIV prevention 
for drug-using women with histories of abuse, but rather a selection of successful interventions that incorporated issues such as empowerment, gender-based inequalities, and history of violence that can be used as models for future interventions. We began the selection process by reviewing HIV interventions listed in the Diffusion of Effective Behavioral Interventions project and best-evidence HIV behavioral interventions by the Centers for Disease Control and Prevention. We then selected additional HIV prevention interventions through electronic searches using PubMed and PsychInfo. The following search terms were used: "intervention", “drug-using”, "women”, "intimate partner violence", "HIV", "HIV/STI", "substance use", and "substance abuse". The selected interventions are presented below and summarized in Table 2 .

\section{Relapse prevention and relationship safety}

Guided by empowerment and social cognitive theories, the relapse prevention and relationship safety (RPRS) intervention takes a synergistic approach of integrating relapse and IPV prevention strategies to address the concurrent goals of establishing relationship safety and reducing drug use. The RPRS is a group-based intervention consisting of 11 group sessions and one individual session for drug-using, abused women in methadone maintenance treatment programs. ${ }^{55}$ The RPRS is tailored to the realities of low-income, urban, African American, and Latina women, and focuses on the enhancement of positive evaluations of self-worth, ethnic pride, and risk avoidance as an investment in the future of their communities. Materials and exercises incorporate social cognitive skill building (ie, coping strategies, boundary setting, negotiation and communication skills) and involve games, brainstorming, role-playing, and small group discussions that build cohesion. Specific to IPV, the RPRS intervention was designed to work with abused women toward harm-free relationships, whether women chose to stay with or leave their partners. Facilitators assessed with each participant her level of potential danger from IPV to ensure that adoption of negotiation and boundary-setting skills was appropriate to her individual situation and would not jeopardize her safety. ${ }^{55}$ The RPRS was compared with an informational condition which consisted of a one-hour didactic presentation of a wide range of local community services (ie, employment services, job training, housing, domestic violence programs, legal services, mental health services) that women in methadone maintenance treatment programs can access, tips on help-seeking, and a comprehensive directory of local IPV-related services. ${ }^{55}$
Compared with informational control participants, RPRS participants were more likely to report a decrease in minor physical or sexual IPV, and minor and severe psychological IPV at the 3-month follow-up. RPRS participants were also more likely than informational control participants to report a decrease in any drug use at 3 months. This pilot randomized controlled trial suggested that a synergistic approach to address the co-occurrence of IPV and continued drug use may be effective in prevention relapse and future violence among drug-involved, abused women in methadone maintenance treatment programs. ${ }^{55}$

\section{Safer Sex Skills Building}

The Safer Sex Skills Building (SSB) intervention was assessed for effectiveness in a sample of women in community drug treatment programs distributed geographically across nine urban and rural US states. ${ }^{56}$ Guided by the Social Cognitive Learning and Empowerment theories, the original version of the intervention, called Project WORTH, was updated to place more emphasis on women's negotiation skills around safer sex and safeguards against the risk of partner abuse as a potential result of assertiveness around safer sex. The SSB intervention is a femalespecific, five-session group intervention emphasizing risk reduction skills, female-male control issues, and negotiation of condom use. The SSB intervention is designed to build cognitive, affective, and behavioral skills for safer sexual decision-making and behavior through active problem-solving, behavioral modeling, role play rehearsal, interval practice, troubleshooting, and peer feedback and support. In this population, more emphasis was placed on women's negotiation skills around safer sex and safeguards against the risk of partner abuse as the potential result of assertiveness around safer sex. The SSB intervention was compared with an HIV/STD education control intervention that focused on providing HIV/STD knowledge, treatment and prevention. ${ }^{56}$

Both the SSB intervention and the HIV/STD education control condition reduced unprotected sex acts at 3-month follow-up. At 6 months after the intervention, the SSB and control conditions significantly differed in change in unprotected sex acts from 3-6-month follow-up. Unprotected sex returned to baseline level in the control condition, while the reductions in high-risk sex were sustained, and even further decreased among women who received the SSB intervention. Women in SSB had 29\% fewer unprotected sex acts than those in the control condition. This skills building intervention that integrated negotiation skills and safeguards 
of partner abuse produced ongoing sexual risk reduction in women in community drug treatment. ${ }^{56}$

\section{Women's Health CoOp}

The North Carolina Women's Health CoOp, used a threegroup randomized design to compare the effects of a personalized woman-focused intervention tailored to gender and culture for out-of-drug-treatment African American women who use crack with the effects of an equal dose intervention based on the revised National Institute on Drug Abuse standard intervention and a delayed-treatment control group. ${ }^{26}$ Although the intervention did not solely include crack users with histories of violence and originally was not designed to address violence, future adaptations in South Africa informed future US-based efforts to include violence and victimization. The woman-focused intervention included culturally enriched content that was grounded in empowerment theory and African-American feminism. The intervention addressed drug dependence as a form of "bondage" and was designed to facilitate greater independence and increase personal power and control over behavior choices as well as life circumstances. ${ }^{26}$ Both the woman-focused intervention and the standard intervention consisted of two individual sessions and two group sessions. The two individual sessions in both intervention groups conducted pretest and post-test HIV counseling. The woman-focused intervention individual sessions also integrated personalized feedback and action plans to address the multidimensional risks of drug use, sexual risk behaviors, and contextual goals to address barriers of education, employment, housing, and parenting (eg, poverty, violence); group sessions used a support-based format to help women understand how they are affected by the multiple contextual influences in their lives and to teach skills to reduce risk and increase a sense of power. ${ }^{26,57}$ The standard intervention was similar to the woman-focused intervention in educational content; however it did not incorporate the gender-specific or culture-specific empowerment approach to develop one's life and change social contexts. ${ }^{57}$

Women in the standard or woman-focused intervention demonstrated significant reductions in crack use and sexual risk behaviors at 3 and 6 months. In addition, at 3-month follow-up, compared with the standard intervention group, women in the woman-focused intervention group showed greater improvement in employment and housing status. At 6-month follow-up, compared with women in the control group, women in the woman-focused intervention group were least likely to engage in unprotected sex. Although both the standard and woman-focused intervention groups demonstrated reductions in sexual risk behaviors, a genderspecific and culturally tailored intervention may be more effective than a standard intervention at reducing sexual risk over time. Moreover, empowerment-based interventions tailored to develop concrete solutions within personal social contexts, more than standard interventions, can influence other life changes (eg, employment, housing) that facilitate independence for African American women. ${ }^{26}$ The Women's Health CoOp has since been designated a Centers for Disease Control and Prevention "best-evidence" HIV behavioral prevention intervention and adapted and shown to be feasible in other at-risk US populations including African American pregnant women in substance abuse treatment, some of whom have histories of violence. ${ }^{57-59}$

This Women's Health CoOp program, designed in the US has also been adapted for international at-risk populations such as South African Black drug-using sex workers. ${ }^{58}$ The adaptation process was informed by the target population and community advisory members. In Pretoria, South Africa, the woman-focused intervention was culturally specific, addressing male dominance and attitudes towards women, multiple partners and beliefs and values about sex, and safer sex practices particularly with boyfriends. ${ }^{60} \mathrm{HIV}$ education was tailored to increase factual knowledge and dispel myths about HIV/AIDS and sexual practices prevalent in this setting (eg, sex with a virgin does not eliminate HIV infection, two male condoms are not better than one). The woman focused intervention also placed an emphasis on contextual issues relevant to sex work (eg, sex-related violence, cultural barriers to increased condom use). In addition to the personalized assessment of women's drug and sexual risks provided in the US-based woman-focused intervention, the South African-based intervention consisted of knowledge of violence prevention strategies such as staying sober to assess the situation, communication techniques in difficult situations, ways to exit a volatile situation, and seeking community resources. At the one-month follow-up, findings showed decreases in the proportion of women reporting unprotected sex and daily use of alcohol and cocaine. Specifically, women in the intervention group increased the use of male condoms with boyfriends and women in both the intervention and standard groups increased their use of female condoms. Daily alcohol and cocaine use also decreased more for women in the woman-focused intervention group. Violence was also assessed at follow-up; although still highly prevalent, women in the woman-focused intervention group were victimized less often than women in the standard intervention group. ${ }^{60}$ The Women's Health 
CoOp was further adapted for substance-using black and colored women in Cape Town, South Africa. ${ }^{61}$

\section{Health Intervention Project}

Although the Health Intervention Project was not designed for drug-using women with histories of violence, the intervention integrated gender-based inequalities. The target population was African American women who were active injection drug users residing in inner city neighborhoods in Atlanta, Georgia. Using a randomized block design, women were assigned to one of three intervention conditions, ie, a four-session enhanced motivation intervention, a foursession enhanced negotiation intervention, and a National Institute on Drug Abuse standard intervention for drug users. ${ }^{62,63}$ The enhanced intervention content was empirically as well as theoretically based, using theories such as social cognitive theory, the theory of reasoned action, the theory of planned behavior, the transtheoretical model of change, and the Theory of Gender and Power. Central to the enhanced intervention conditions were the social context of the women's daily lives, including the meaning of behaviors and social interactions, gender dynamics, economic stressors, gender-specific norms and values, and power and control. The enhanced motivation condition focused on identifying what each woman would be motivated to change in her life and the negotiation condition focused on specific skills-training of condom use and safe injection practices, gaining control, developing assertiveness, and conflict resolution. The standard condition focused on HIV knowledge including HIV risk and protective behaviors. The enhanced conditions were more effective than the National Institute on Drug Abuse standard condition at reducing the number of paying partners and the frequency of sex with paying partners (negotiation condition), and the use of crack in risky settings (motivation condition). Components of both interventions may prove useful for reducing the risk of HIV infection among similar populations of women. ${ }^{62}$

\section{Women Fighting Infection Together}

The Women Fighting Infection Together (Women FIT) intervention integrated a body empowerment approach for female out-of-treatment drug users in three US cities (in Providence, New York, and Philadelphia). ${ }^{64}$ In addition to a framework that examined gender-based power imbalances in heterosexual relationships, a key focus of the Women FIT intervention was increasing knowledge about, confidence in, and a sense of ownership of the woman's body, especially her reproductive organs. These desired effects were thought to be mediated by promotion of female-initiated barrier methods, such as the female condom, and the use of peer counseling and participatory sessions to augment the empowerment process. Four group sessions were led by peers and encouraged participation and exchange among the women to build solidarity among women as a means to confront the collective experience of economic stress and poor health emanating from their low status in a patriarchal society, effects greatly exaggerated in the drug-using community. Group session 1 sought to increase knowledge of the female reproductive anatomy, knowledge of the risks of HIV infection, and familiarity and comfort level with one's genitals and reproductive anatomy, required for first attempts at various barrier protection methods (such as the female condom). Group session 2 was centered around role-playing and sought to contextualize the skills and knowledge from the first session, by elaborating on how HIV/STD protection methods might be used in various scenarios (eg, with main versus paying partners, sex in the context of substance use). Group session 3 focused on women's bodies and health needs, emphasizing group members' own roles as advocates in their own health care, including their reproductive health. Group session 4 focused on strategies for reducing or avoiding violence, abuse, and violent sex (eg, physical self-defense techniques, community support). ${ }^{64}$

The Women FIT intervention has only been tested for its feasibility. ${ }^{64}$ Women assigned to the intervention group demonstrated greater short-term knowledge increases regarding the body and reproductive tract, as well as practical knowledge about the different protection methods offered, when compared with women in the control group. Women in the intervention group also reported trying the protection methods offered over the 2-month period, including making requests to the study team for clinical referrals to obtain the prescription methods (eg, diaphragm, cervical cap). The sense of solidarity achieved in the group interactions and the quality of the peer communications were also strongly endorsed by the women. ${ }^{64}$

\section{Boston Consortium Model}

The Boston Consortium Model ${ }^{65}$ is an HIV prevention intervention for ethnically diverse, drug-using women with co-occurring psychiatric and physical and/or sexual abuse histories. It was part of the SAMHSA-funded Women, Co-Occurring Disorders and Violence Study and is listed in the National Registry of Evidence-Based Programs and Practices. ${ }^{66}$ The study assessed HIV risk behaviors, mental health, trauma, and substance abuse outcomes among women 
receiving the Boston Consortium Model $^{65}$ and those receiving substance abuse services as usual.

The intervention integrated assessment and treatment planning for trauma history and addiction disorders. ${ }^{65}$ In addition, the trauma-specific intervention group provided skill building in the areas of economics, family reunification, and leadership. Women who received the integrated trauma treatment intervention were more likely to demonstrate reduced sexual risk behaviors relative to the control group at 6 and 12 months follow-up. ${ }^{67}$ In addition, women in the intervention had a higher relationship power at 6 and 12 months, and in turn were less likely to engage in unprotected sex than women with lower relationship power scores. The intervention also produced significantly greater reductions in trauma and mental health symptomatology and drug abstinence. ${ }^{67}$

The Boston Consortium Model illustrates the value of integrated interventions that address gender-based violence, history of abuse, mental health, and drug abuse in the context of HIV prevention research. As a whole, these interventions provide evidence that it is important to account for genderbased inequalities in the design of HIV prevention interventions for drug-using women.

\section{Discussion}

The accumulating evidence linking the epidemics of HIV, substance abuse, and partner violence underscores the need to address these issues synergistically. To date, there is a paucity of HIV prevention interventions designed for drug-using women with histories of partner violence. The few existing interventions have targeted both women in drug treatment programs and those out of treatment and were theory-based, often guided by empowerment theory, and combined cognitive behavioral components. The RPRS intervention demonstrated the importance of addressing skills specific to handling partner violence, including assessment of personal risk, safety planning, negotiation skills, and boundary-setting. On the other hand, the SSB intervention and Health Intervention Project were successful in integrating gender-based inequalities including male dominance, gender-specific norms, and power and control. The Boston Consortium Model, Health Intervention Project, Women FIT, and Women's Health CoOp emphasized the importance of integrating economic barriers (ie, education, employment, housing) into interventions, in efforts to address underlying determinants to facilitate reductions in HIV risk behaviors. No single intervention addressed all of these issues; rather the focus was on one or none of these issues. With the exception of the Boston Consortium Model and RPRS, the interventions promoted the use of the female condom, a biomedical approach representative of female-initiated barrier methods. Regardless of the geographic setting, most of these interventions were culturally tailored to the target population, often as a result of direct involvement from members of the target population and community during intervention development and implementation. This is promising in light of increasing emphasis on the need for culture-specific HIV prevention interventions for at-risk populations including drug-using women. ${ }^{34,58,68-72}$ However, HIV prevention interventions for drug-using women with histories of partner violence are not widespread, and do not account for components of the HIV risk environment. Moreover, biomedical approaches such as use of microbicides, have yet to be assessed for feasibility in this at-risk population.

To address HIV risk among drug-using women with histories of partner violence, interventions should target individuals, men, couples, and social networks. HIV prevention interventions for drug-using women with histories of partner violence that focus on the physical, social, economic, and political environments that influence HIV risk-taking among drug-using women with histories of violence are significantly more likely to impact the incidence of HIV across larger population groups. ${ }^{4}$ The Women's Health CoOp intervention in North Carolina addresses some of these underlying determinants (eg, employment, housing, poverty). This reinforces the need for HIV prevention interventions to move beyond specific risk groups to address the micro-level and macrolevel determinants that shape their risk environments. ${ }^{4} \mathrm{HIV}$ prevention interventions for drug-using women with histories of partner violence should be targeted to not only women but also men. Masculinity norms and male psychological dominance are related to men's sexual risk behaviors ${ }^{73-75}$ and perpetration of IPV. ${ }^{76,77}$ To change masculinity and social norms toward violence against women, it is important to raise men's awareness of the scope of violence against women, the multiple ways in which it contributes to HIV risk for drug users, and the resources available for partner violence and HIV. And, although some evidence suggests that couple-based HIV prevention interventions may not work as well for couples experiencing severe conflict or partner violence, research has yet to identify whether the effects of couple-based interventions are moderated definitively by gender-based inequalities such as partner violence and relationship power differentials. ${ }^{78}$ It has been considered that couple-based HIV prevention interventions may provide the opportunity for a more open discussion between partners on sensitive topics, such as power imbalances associated with 
sexual coercion, the inability to negotiate condom use, and gender inequalities in needle sharing. ${ }^{5}$

HIV prevention experts agree that no single prevention strategy will be $100 \%$ effective in reducing new infections, nor acceptable and applicable to all populations. ${ }^{79}$ Theory-driven behavioral interventions will be a necessary, but not singularly sufficient, component of a successful prevention program. A number of biomedical approaches, such as pre-exposure prophylaxis and microbicides, have been shown to have a significant impact on curbing new HIV infections in vulnerable women. Combination HIV prevention consisting of evidence-based behavioral, biomedical, and interventions that incorporate the risk environment must be developed to be appropriate, acceptable, and deliverable to populations with high levels of coverage and adherence. ${ }^{79}$ Addressing multiple determinants affords the opportunity to ameliorate the conditions underpinning increased risk of HIV as well as structural change. ${ }^{14}$ For example, targeting employment and housing opportunities in conjunction with substance abuse and mental health counseling may prevent women's exposure to the vulnerabilities of exchanging sex for money, drugs, or other resources, in turn reducing the risk for HIV acquisition. Given the limited amount of time and resources available for delivery of interventions, we need research that allows us to prioritize the order in which to address these issues. Although it is impossible to address the entire risk environment, select components can be integrated through estimation of the potential effect size and population attributable fraction, identification of possible synergies and antagonism among the determinants and other intervention components (eg, biomedical, behavioral), and assessment of feasibility, scale-up, and evaluation of the combination HIV prevention intervention. ${ }^{79}$ This effort is currently underway with a combination prevention approach for alcohol and drugusing women in South Africa, some of whom are affected by gender-based violence. ${ }^{80}$ If effective, this intervention may serve as an example for integrated HIV prevention interventions in South Africa.

However, there remains a critical need to develop targeted combination HIV prevention interventions for drug-using women with histories of partner violence in other geographic regions. Comprehensive combination HIV interventions will be those that address risk factors at the individual, dyadic, social, economic, and political levels, while accounting for the stage of the HIV epidemic for drug-using women in a particular region. Effective interventions will be those that fail to neglect gender, culture, and ethnicity. Ultimately, this inclusive approach will have a significant impact on reducing
HIV infections among drug-using women with histories of partner violence.

\section{Acknowledgments}

This work was supported by the National Institutes of Health (K01DA031593, L60MD003701, R25DA025571, and R25MH080664 to JKS and K01DA026307 to MDU). The authors also thank Thomas Patterson, Steffanie Strathdee, and Anita Raj from the University of California, San Diego. The contents of this article are solely the responsibility of the authors and do not necessarily represent the official views of the National Institutes of Health.

\section{Disclosure}

The authors report no conflicts of interest in this work.

\section{References}

1. Joint United Nations Programme on HIV/AIDS (UNAIDS). UNAIDS Report on the Global AIDS Epidemic. 2010.

2. Prejean J, Song R, Hernandez A, et al. Estimated HIV incidence in the United States, 2006-2009. PLoS One. 2011;6(8):e17502.

3. Mathers BM, Degenhardt L, Phillips B, et al. Global epidemiology of injecting drug use and HIV among people who inject drugs: A systematic review. Lancet. 2008;372(9651):1733-1745.

4. Stockman JK, Strathdee SA. HIV among people who use drugs: A global perspective of populations at risk. $J$ Acquir Immune Defic Syndr. 2010;55 Supp1 1:S17-S22.

5. El-Bassel N, Terlikbaeva A, Pinkham S. HIV and women who use drugs: Double neglect, double risk. Lancet. 2010;376(9738):312-314.

6. Wechsberg WM, Luseno W, Riehman K, Karg R, Browne F, Parry C. Substance use and sexual risk within the context of gender inequality in South Africa. Subst Use Misuse. 2008;43(8-9):1186-1201.

7. Ulibarri MD, Sumner LA, Cyriac A, Amaro H. Power, violence and HIV risk in women. In: Paludi M, Denmark FL, editors. Victims of Sexual Assault and Abuse: Resources and Responses for Individuals and Families: Vol. 1. Incidence and Psychological Dimensions. Santa Barbara, CA: Praeger; 2010.

8. El-Bassel N, Gilbert L, Witte S, Wu E, Chang M. Intimate partner violence and HIV among drug-involved women: Contexts linking these two epidemics - challenges and implications for prevention and treatment. Subst Use Misuse. 2011;46(2-3):295-306.

9. El-Bassel N, Gilbert L, Wu E, Go H, Hill J. HIV and intimate partner violence among methadone-maintained women in New York City. Soc Sci Med. 2005;61(1):171-183.

10. Panchanadeswaran S, Frye V, Nandi V, Galea S, Vlahov D, Ompad D. Intimate partner violence and consistent condom use among drug-using heterosexual women in New York City. Women Health. 2010;50(2): $107-124$.

11. Shannon K, Kerr T, Bright V, Gibson K, Tyndall MW. Drug sharing with clients as a risk marker for increased violence and sexual and drug-related harms among survival sex workers. AIDS Care. 2008; 20(2):228-234.

12. Shannon K, Kerr T, Allinott S, Chettiar J, Shoveller J, Tyndall MW. Social and structural violence and power relations in mitigating HIV risk of drug-using women in survival sex work. Soc Sci Med. 2008; 66(4):911-921.

13. El-Bassel N, Gilbert L, Wu E, Go H, Hill J. Relationship between drug abuse and intimate partner violence: A longitudinal study among women receiving methadone. Am J Public Health. 2005;95(3):465-470.

14. Rhodes T, Simic M. Transition and the HIV risk environment. $B M J$. 2005;331(7510):220-223. 
15. Strathdee SA, Hallett TB, Bobrova N, et al. HIV and risk environment for injecting drug users: the past, present, and future. Lancet. 2010; 376(9737):268-284.

16. Connell R. Gender and Power. Stanford, CA: Stanford University Press; 1987.

17. Wingood GM, DiClemente RJ. Application of the theory of gender and power to examine HIV-related exposures, risk factors, and effective interventions for women. Health Educ Behav. 2000;27(5):539-565.

18. Andrade R, Estrada AL. Are Hispana IDUs Tecatas?: Reconsidering gender and culture in Hispana injection drug use. Subst Use Misuse. 2003;38(8):1133-1158.

19. Suffet F, Brotman R. Female drug use: Some observations. Int JAddict. 1976;11(1):19-33.

20. Cheng WS, Garfein RS, Semple SJ, Strathdee SA, Zians JK, Patterson TL. Differences in sexual risk behaviors among male and female HIV-seronegative heterosexual methamphetamine users. Am J Drug Alcohol Abuse. 2009;35(5):295-300.

21. St Lawrence JS, Wilson TE, Eldridge GD, Brasfield TL, O'Bannon RE 3rd. Community-based interventions to reduce low income, African American women's risk of sexually transmitted diseases: A randomized controlled trial of three theoretical models. Am J Community Psychol. 2001;29(6):937-964.

22. Rappaport J. Studies in empowerment: Introduction to the issues. In: Rappaport J, Swift C, Hess R, editors. Studies in Empowerment: Steps Toward Understanding and Action. New York, NY: The Haworth Press; 1984.

23. Wallerstein N. Powerlessness, empowerment and health: Implications for health promotion programs. Am J Health Promot. 1992;6(3): 197-205.

24. Zimmerman M, Warschausky S. Empowerment theory for rehabilitation research: Conceptual and methodological issues. Rehabil Psychol. 1998;43(1):3-16.

25. Zimmerman M. Empowerment theory: Psychological, organizational and community levels of analysis. In: Rappaport J, Seidman E, editors. Handbook of Community Psychology. Dordrecht, Netherlands: Kluwer Academic Publishers; 2000.

26. Wechsberg WM, Lam WK, Zule WA, Bobashev G. Efficacy of a woman-focused intervention to reduce HIV risk and increase selfsufficiency among African American crack abusers. Am J Public Health. 2004;94(7):1165-1173.

27. Perkins DD, Zimmerman MA. Empowerment theory, research, and application. Am J Community Psychol. 1995;23(5):569-579.

28. Sherman SG, German D, Cheng Y, Marks M, Bailey-Kloche M. The evaluation of the JEWEL project: An innovative economic enhancement and HIV prevention intervention study targeting drug using women involved in prostitution. AIDS Care. 2006;18(1):1-11.

29. Busch N, Valentine D. Empowerment practice: A focus on battered women. Affilia. 2000;15(1):82-95.

30. Kim JC, Watts CH, Hargreaves JR, et al. Understanding the impact of a microfinance-based intervention on women's empowerment and the reduction of intimate partner violence in South Africa. Am J Public Health. 2007;97(10):1794-1802.

31. Singer M. Dose of drugs, a touch of violence, a case of AIDS: Conceptualizing the SAVA syndemic. Free Inq Creat Sociol. 1996;24(2): 99-110.

32. Centers for Disease Control and Prevention. Syndemics overview history: what is a syndemic? 2008. Available from: http://www.cdc. gov/syndemics/overview-history.htm. Accessed November 16, 2011.

33. Singer M. AIDS and the health crisis of the US urban poor; the perspective of critical medical anthropology. Soc Sci Med. 1994;39(7): 931-948.

34. Gonzalez-Guarda R, Florom-Smith A, Thomas T. A syndemic model of substance abuse, intimate partner violence, HIV infection, and mental health among Hispanics. Public Health Nurs. 2011;28(4):366-378.

35. NIH Office of AIDS Research. FY 2012 Trans-NIH plan for HIV related research - priority: reducing new infections. Bethesda, MD: NIH Office of AIDS Research, US Department of Health and Human Services; 2012.
36. Padian NS, Buve A, Balkus J, Serwadda D, Cates W Jr. Biomedical interventions to prevent HIV infection: evidence, challenges, and way forward. Lancet. 2008;372(9638):585-599.

37. Kelesidis T, Landovitz RJ. Preexposure prophylaxis for HIV prevention. Curr HIV/AIDS Rep. 2011;8(2):94-103.

38. Garcia-Lerma JG, Paxton L, Kilmarx PH, Heneine W. Oral pre-exposure prophylaxis for HIV prevention. Trends Pharmacol Sci. 2010;31(2): 74-81.

39. Abbas UL. Uptake of biomedical interventions for prevention of sexually transmitted HIV. Curr Opin HIV AIDS. 2011;6(2): 114-118.

40. Centers for Disease Control and Prevention. High-impact HIV Prevention: CDC's Approach to Reducing HIV infections in the United States. Atlanta, GA: Centers for Disease Control and Prevention; 2011.

41. Weber J, Desai K, Darbyshire J; the Microbicides Development Programme. The development of vaginal microbicides for the prevention of HIV transmission. PLoS Med. 2005;2(5):e142.

42. Microbicide Trials Network. MTN: Microbicide trials network. 2011. Available from: http://www.mtnstopshiv.org/node/84. Accessed November 16, 2011.

43. Hladik F, Doncel GF. Preventing mucosal HIV transmission with topical microbicides: Challenges and opportunities. Antiviral Res. 2010; 88 Suppl 1:S3-S9.

44. Abdool Karim Q, Abdool Karim SS, Frohlich JA, et al. Effectiveness and safety of tenofovir gel, an antiretroviral microbicide, for the prevention of HIV infection in women. Science. 2010;329:1168-1174.

45. Nuttall J. Microbicides in the prevention of HIV infection: Current status and future directions. Drugs. 2010;70(10):1231-1243.

46. Mason TH, Foster SE, Finlinson HA, et al. Perspectives related to the potential use of vaginal microbicides among drug-involved women: Focus groups in three cities in the United States and Puerto Rico. AIDS Behav. 2003;7(4):339-351.

47. Hammett TM, Norton GD, Mason TH, et al. Drug-involved women as potential users of vaginal microbicides for HIV and STD prevention: A three-city survey. J Womens Health Gend Based Med. 2000; 9(10):1071-1080.

48. Gollub EL. The female condom: Tool for women's empowerment. Am J Public Health. 2000;90(9):1377-1381.

49. Narrigan D. Women's barrier contraceptive methods: Poised for change. J Midwifery Womens Health. 2006;51(6):478-485.

50. Minnis AM, Padian NS. Effectiveness of female controlled barrier methods in preventing sexually transmitted infections and HIV: Current evidence and future research directions. Sex Transm Infect. 2005;81(3):193-200.

51. Hoffman S, Mantell J, Exner T, Stein Z. The future of the female condom. Int Fam Plan Perspect. 2004;30(3):139-145.

52. Surratt HL, Wechsberg WM, Cottler LB, Leukefeld CG, Klein H, Desmond DP. Acceptability of the female condom among women at risk for HIV infection. Am Behav Sci. 1998;41:1157-1170.

53. El-Bassel N, Krishnan SP, Witte S, Schilling RF, Catan V, Pollin S. Correlates of intention to use the female condom among women taking methadone. Womens Health Issues. 1998;8(2):112-122.

54. Gollub EL. A neglected population: Drug-using women and women's methods of HIV/STI prevention. AIDS Educ Prev. 2008; 20(2):107-120.

55. Gilbert L, El-Bassel N, Manuel J, et al. An integrated relapse prevention and relationship safety intervention for women on methadone: Testing short-term effects on intimate partner violence and substance use. Violence Vict. 2006;21(5):657-672.

56. Tross S, Campbell AN, Cohen LR, et al. Effectiveness of HIV/STD sexual risk reduction groups for women in substance abuse treatment programs: Results of NIDA clinical trials network trial. JAcquir Immune Defic Syndr. 2008;48(5):1-9.

57. Wechsberg WM, Novak SP, Zule WA, et al. Sustainability of intervention effects of an evidence-based HIV prevention intervention for African American women who smoke crack cocaine. Drug Alcohol Depend. 2010;109(1-3):205-212. 
58. Wechsberg WM, Browne FA, Ellerson RM, Zule WA. Adapting the evidence-based women's CoOp intervention to prevent human immunodeficiency virus infection in North Carolina and international settings. N C Med J. 2011;71(5):477-481.

59. Jones HE, Berkman ND, Kline TL, et al. Initial feasibility of a woman-focused intervention for pregnant African-American women. Int J Pediatr. 2011;2011:389285.

60. Wechsberg WM, Luseno WK, Lam WK, Parry CD, Morojele NK. Substance use, sexual risk, and violence: HIV prevention intervention with sex workers in Pretoria. AIDS Behav. 2006;10(2):131-137.

61. Wechsberg WM, Luseno WK, Karg RS, et al. Alcohol, cannabis, and methamphetamine use and other risk behaviours among black and coloured South African women: A small randomized trial in the Western Cape. Int J Drug Policy. 2008;19(2):130-139.

62. Sterk CE, Theall KP, Elifson KW. Effectiveness of a risk reduction intervention among African American women who use crack cocaine. AIDS Educ Prev. 2003;15(1):15-32.

63. Sterk CE, Theall KP, Elifson KW, Kidder D. HIV risk reduction among African-American women who inject drugs: A randomized controlled trial. AIDS Behav. 2003;7(1):73-86.

64. Gollub EL, Morrow KM, Mayer KH, et al. Three city feasibility study of a body empowerment and HIV prevention intervention among women with drug use histories: Women FIT. $J$ Womens Health (Larchmt). 2010;19(9):1705-1713.

65. Amaro H, McGraw S, Larson MJ, Lopez L, Nieves R, Marshall B. Boston consortium of services for families in recovery: A traumainformed intervention model for women's alcohol and drug addiction treatment. Alcohol Treat Q. 2004;22:95-119.

66. National Registry of Evidence-Based Programs and Practices, SAMHSA. Boston Consortium Model: Trauma-Informed Substance Abuse Treatment for Women. 2010. Available from: http://nrepp.samhsa.gov/ ViewIntervention.aspx?id=86. Accessed November 16, 2011.

67. Amaro H, Dai J, Arevalo S, et al. Effects of integrated trauma treatment on outcomes in a racially/ethnically diverse sample of women in urban community-based substance abuse treatment. J Urban Health. 2007;84(4):508-522.

68. Williams JK, Wyatt GE, Wingood G. The four Cs of HIV prevention with African Americans: Crisis, condoms, culture, and community. Curr HIV/AIDS Rep. 2010;7(4):185-193.

69. Crepaz N, Marshall KJ, Aupont LW, et al. The efficacy of HIV/ STI behavioral interventions for African American females in the United States: A meta-analysis. Am J Public Health. 2009; 99(11):2069-2078.
70. El-Bassel N, Caldeira NA, Ruglass LM, Gilbert L. Addressing the unique needs of African American women in HIV prevention. Am J Public Health. 2009;99(6):996-1001.

71. Amaro H. Love, sex, and power. considering women's realities in HIV prevention. Am Psychol. 1995;50(6):437-447.

72. Amaro H, Raj A, Vega RR, Mangione TW, Perez LN. Racial/ethnic disparities in the HIV and substance abuse epidemics: Communities responding to the need. Public Health Rep. 2001;116(5):434-448.

73. El-Bassel N, Gilbert L, Golder S, et al. Deconstructing the relationship between intimate partner violence and sexual HIV risk among drug-involved men and their female partners. AIDS Behav. 2004;8(4):429-439.

74. Decker MR, Seage GR 3rd, Hemenway D, Gupta J, Raj A, Silverman JG. Intimate partner violence perpetration, standard and gendered STI/HIV risk behaviour, and STI/HIV diagnosis among a clinic-based sample of men. Sex Transm Infect. 2009;85(7):555-560.

75. Santana MC, Raj A, Decker MR, La Marche A, Silverman JG. Masculine gender roles associated with increased sexual risk and intimate partner violence perpetration among young adult men. $J$ Urban Health. 2006;83(4):575-585.

76. Raj A, Santana MC, La Marche A, Amaro H, Cranston K, Silverman JG. Perpetration of intimate partner violence associated with sexual risk behaviors among young adult men. Am J Public Health. 2006;96(10):1873-1878.

77. Frye V, Latka MH, Wu Y, et al. Intimate partner violence perpetration against main female partners among HIV-positive male injection drug users. J Acquir Immune Defic Syndr. 2007;46 Suppl 2:S101-S109.

78. El-Bassel N, Gilbert L, Witte S, Wu E, Hunt T, Remien RH Couple-based HIV prevention in the United States: Advantages, gaps, and future directions. J Acquir Immune Defic Syndr. 2010;55 Suppl 2: S98-S101.

79. Kurth AE, Celum C, Baeten JM, Vermund SH, Wasserheit JN. Combination HIV prevention: Significance, challenges, and opportunities. Curr HIV/AIDS Rep. 2011;8(1):62-72.

80. US Department of Health and Human Services. Combination prevention for vulnerable women in South Africa. PI: Wechsberg, Wendee M. 2011. Available from: http://projectreporter.nih.gov/project_info_description. cfm?aid=8189313\&icde=0. Accessed November 16, 2011.
Substance Abuse and Rehabilitation

\section{Publish your work in this journal}

Substance Abuse and Rehabilitation is an international, peer-reviewed, open access journal publishing original research, case reports, editorials, reviews and commentaries on all areas of addiction and substance abuse and options for treatment and rehabilitation. The manuscript management system is completely online and includes a very quick and fair

\section{Dovepress}

peer-review system. Visit http://www.dovepress.com/testimonials.php to read real quotes from published authors. 